\title{
PREVALENCE OF LOWER URINARY TRACT INFECTIONS IN WOMEN ATTENDING GYNAECOLOGY OPD, AT A TERTIARY CARE HOSPITAL IN EASTERN INDIA
}

\author{
Poonam ${ }^{1}$, Tarun Kumar ${ }^{2}$
}

${ }^{1}$ Associate Professor, Department of Obstetrics and Gynaecology, Indira Gandhi Institute of Medical Sciences (IGIMS), Patna. ${ }^{2}$ Additional Professor, Department of Physiology, Indira Gandhi Institute of Medical Sciences (IGIMS), Patna.

\section{ABSTRACT}

\section{BACKGROUND}

Urinary Tract Infections (UTIs) in women are amongst the most common infections in the outpatient population and have a negative impact on the quality of life. The overall prevalence of lower urinary tract symptoms amongst women is $67 \%$. In fact, the prevalence of LUTS in them is reportedly higher than many other chronic diseases. ${ }^{1,2}$ Nearly 1 in 3 women will have at least one episode of UTI requiring antimicrobial therapy by the age of 24 years. Almost half of all women will experience one UTI during their lifetime. ${ }^{3}$

\section{MATERIALS AND METHODS}

It was a retrospective study, carried out in the Department of Obstetrics and Gynaecology at a tertiary care centre, in eastern India. All patients attending gynaecology OPD between April 2014 and March 2015 were included in the study.

\section{RESULTS}

In this study, the highest prevalence of urinary tract infection was observed in women of reproductive age group (31.3\%). The symptoms were least common (1.6\%) in older women (60 - 70). The strongest, modifiable behavioural risk factor is the frequency of sexual intercourse. However, some forms of contraception also increase the risk of UTI in some women. Majority amongst those with LUTS experienced urgency $(34 \%, n=637)$ with $26 \%(n=487)$ having increased frequency.

\section{CONCLUSION}

Therefore, studying women's LUTS may be of help in giving proper intervention resources to improve individual's quality of life and also to provide preventive care in the future.

\section{KEYWORDS}

Dysuria, Incontinence, Recurrent UTI, Urinary Retention, Urinary Tract Infection.

HOW TO CITE THIS ARTICLE: Poonam, Kumar T. Prevalence of lower urinary tract infections in women attending Gynaecology OPD at a tertiary care hospital in eastern India. J. Evolution Med. Dent. Sci. 2017;6(30):2432-2435, DOI: 10.14260/Jemds/2017/525

\section{BACKGROUND}

Urinary Tract Infections (UTIs) in women are amongst the most common infections in the outpatient population and have a negative impact on the quality of life. The overall prevalence of lower urinary tract symptoms amongst women is $67 \%$. In fact, the prevalence of LUTS in them is reportedly higher than many other chronic diseases. ${ }^{1,2}$ Nearly, 1 in 3 women will have at least one episode of UTI requiring antimicrobial therapy by the age of 24 years. Almost half of all women will experience one UTI during their lifetime. ${ }^{3}$

This study was done to know the prevalence of urinary tract infections amongst women attending gynaecology OPD at a tertiary care hospital and also to identify the associated risk factors, so that advice could be given to reduce the incidence.

\section{MATERIALS AND METHODS}

It was a retrospective study, carried out in the Department of Obstetrics and Gynaecology at a tertiary care hospital, in

Financial or Other, Competing Interest: None.

Submission 08-03-2017, Peer Review 01-04-2017,

Acceptance 07-04-2017, Published 13-04-2017.

Corresponding Author:

Dr. Poonam,

Doctors' Qr. No. D-6/1,

IGIMS Campus, Patna-14, Bihar.

E-mail:drpoonam8@yahoo.com

DOI: $10.14260 /$ jemds $/ 2017 / 525$

\section{(c) $(1) \ominus$}

eastern India. All patients attending gynaecology OPD between April 2014 and March 2015 were included in the study. Data was retrieved and analysed using the case sheets and documented lab reports from OPD files. Patients referred from other departments and lab tests done outside the Institute were also considered in this study.

For the purpose of this study (besides positive urine culture), positive UTI was defined as the presence of two of the following three criteria on presentation or urinalysis: leukocyte esterase, nitrites or a documented complaint of dysuria. ${ }^{4}$ These criteria are consistent with clinical findings associated with a high predictive value for UTI with each additional criterion making UTI more likely. ${ }^{5}$

For the purpose of this study, terms defined were as follows;

Recurrent UTI was defined by two or more urine infections in six months or greater than three infections in one year.

Urinary urgency is the complaint of a sudden compelling desire to pass urine, which is difficult to defer and urge incontinence is the complaint of involuntary leakage, accompanied by or immediately preceded by urgency.

Stress urinary incontinence was involuntary leakage of urine on effort, exertion, sneezing or coughing. ${ }^{6}$

\section{Aims and Objective}

To know the prevalence of urinary tract infections amongst patients attending Gynaecology OPD and to identify the 
associated risk factors, so that advice could be given to reduce the incidence.

\section{RESULTS}

Data was collected from the OPD files for the specified period. The total number of cases reviewed was 5651. Out of this, 1884 were new and 3767 were old patients.

\begin{tabular}{|c|c|c|c|c|}
\hline Age & $\begin{array}{c}\text { No. of } \\
\text { patients } \\
\text { seen in } \\
\text { Gynaecology } \\
\text { OPD }\end{array}$ & $\begin{array}{c}\text { Percentage } \\
\text { of total } \\
\text { patients } \\
\text { seen }\end{array}$ & $\begin{array}{c}\text { No. of } \\
\text { patients with } \\
\text { lower } \\
\text { urinary } \\
\text { tract } \\
\text { symptoms }\end{array}$ & $\%$ \\
\hline $19-29$ & 678 & $12 \%$ & 120 & 6.4 \\
\hline $30-39$ & 1808 & $32 \%$ & 855 & 45.6 \\
\hline $40-49$ & 1413 & $25 \%$ & 570 & 30.4 \\
\hline $50-59$ & 1243 & $22 \%$ & 300 & 16 \\
\hline $60-70$ & 509 & $9 \%$ & 30 & 1.6 \\
\hline Total & $\mathbf{5 6 5 1}$ & $\mathbf{1 0 0}$ & $\mathbf{1 8 7 5}$ & $\mathbf{3 3 . 1 7}$ \\
\hline \multicolumn{4}{|r|}{ Table 1. Age Wise Distribution of Patients } \\
\hline
\end{tabular}

\begin{tabular}{|c|c|c|}
\hline Associated Complaints & No. of Patients & $\mathbf{\%}$ \\
\hline Menorrhagia & 357 & $19 \%$ \\
\hline Dysmenorrhoea & 330 & $17.6 \%$ \\
\hline Polymenorrhoea & 244 & $13 \%$ \\
\hline Vaginal Discharge & 243 & $13 \%$ \\
\hline Constipation & 412 & $21.9 \%$ \\
\hline Pelvic Mass & 6 & $0.32 \%$ \\
\hline Prolapse & 13 & $0.6 \%$ \\
\hline Table 2. Main Pathological Conditions and Diseases \\
Presenting with Urinary Symptoms \\
\hline
\end{tabular}

\begin{tabular}{|c|c|c|}
\hline Urinary Symptoms & No. of Patients & \% \\
\hline Urgency & 637 & 34 \\
\hline Nocturia & 356 & 19 \\
\hline Urinary incontinence & 375 & 20 \\
- Stress incontinence & 318 & 17 \\
- Urge incontinence & 3 & 0.1 \\
- True incontinence & 487 & 26 \\
\hline Frequency & 8 & 0.4 \\
\hline Urinary retention & 93 & 5 \\
\hline Intermittence & 131 & 7 \\
\hline Haematuria & 487 & 26 \\
\hline Recurrent UTI & 375 & 20 \\
\hline \multicolumn{2}{|c|}{ Tysuria } & \\
\hline \multicolumn{2}{|c|}{ Table 3. Urinary Symptoms } \\
\hline
\end{tabular}

Note- the number of subjects do not match the symptoms, as there were more than one lower urinary tract symptoms in some patients.

\section{DISCUSSION}

During the time period April 2014 - March 2015, five thousand seven hundred patients were seen in the Department of Gynaecology. Five thousand six hundred and fifty one were registered for OPD and the rest forty nine were emergency admissions.

In this study, the overall prevalence of lower urinary tract symptoms was considerable, i.e. $31.3 \%$.

This figure is consistent with a previous study reporting similar rate, i.e. $31.5 \%$ by Savitha T et al. ${ }^{7}$

However, there are wide variations in the prevalence of lower urinary tract infections. Rate as high as $66.78 \%$ has been quoted by Ramesh et al. ${ }^{8} \mathrm{~A}$ reason for such wide variation in these estimates could be the use of selected groups of women, eg. pregnancy, elderly women, young women, etc. in their study. Earlier research demonstrated that despite the presence of symptoms, women do not immediately seek medical intervention for UTI. ${ }^{9}$

Majority of patients were in the reproductive age group. Those between 30 - 39 years were maximum in number $(32 \%, \mathrm{n}=1808)$ followed by $40-49$ years' age group $(25 \%$, $\mathrm{n}=1413$ ) with LUTS in $45.6 \%$ and $30.4 \%$ of them respectively. Twenty two percent $(n=1243)$ of the study population were between 50 - 59 years with 300 of them i.e. 16\% having LUTS. Only $9 \%$ (505) constituted the $60-70$ years' age group, of which only 30 patients (i.e. 1.6\%) had lower urinary tract symptoms.

In this study, the highest prevalence of urinary tract infection was observed in women of reproductive age group. This could possibly be due to the large number of patients registered in this group. The symptoms were least common $(1.6 \%)$ in older women $(60-70)$, consistent with the findings of Jolley et al. ${ }^{10}$

A wide range of factors have been identified that can increase the susceptibility to UTI.

Biological factors that have been identified include congenital abnormalities, 11 the presence of a urinary obstruction ${ }^{12}$ and a prior history of UTI.11

The strongest, modifiable behavioural risk factor is the frequency of sexual intercourse. Sexual activity can move microbes from the bowel or vaginal cavity to the urethral opening. If these microbes have special characteristics that allow them to live in the urinary tract, it is harder for the body to remove them quickly enough to prevent infection. So following sexual intercourse, most women have a significant number of bacteria in their urine, but the body normally clears them within 24 hours. Hence, the importance of urination shortly after sex.

However, some forms of contraception also increase the risk of UTI in some women. The use of spermicides and condoms may irritate and traumatise the tissues during sexual activity. ${ }^{13}$ Thus, increasing the risk of bacterial invasion of the affected and surrounding tissues. Switching to lubricated condoms without spermicide or using a nonspermicidal lubricant may help prevent UTIs.

In younger patients, a history of maternal UTI and age at first UTI are also important risk factors, suggesting a genetic component to susceptibility. The non-secretor status or ABO blood group antigens are known to increase susceptibility. ${ }^{14}$ In our study, $6.4 \%(n=120)$ patients with lower urinary tract symptoms belonged to 19 - 29 years' age group.

Altogether, 1875 patients presented with lower urinary tract symptoms.

Eight hundred and seventy patients, i.e. $46.4 \%$ of the total patients with lower urinary tract symptoms had positive urine culture. The most common organism isolated in these patients was E. coli (70\%) followed by Staphylococcus saprophyticus and Klebsiella pneumonia.

As the results from this study revealed that the important infecting organisms were commensals of perianal and vaginal regions, this calls for increase in personal hygiene also.

Moreover, $60 \%$ of those with lower urinary tract symptoms belonged to lower socioeconomic group where 
homemade, reusable sanitary pads are commonly used. This again calls for maintaining menstrual hygiene.

Many subjects presented with more than one lower urinary tract symptoms.

Majority amongst those with Lower Urinary Tract Symptoms (LUTS) experienced urgency $(34 \%, \mathrm{n}=637)$ with $26 \%(n=487)$ having increased frequency.

Dysuria, which is the characteristic primary symptom in adult, accounted for $20 \%(n=375)$ of cases. In this, sensation of bladder fullness or lower abdominal discomfort is usually present.

Nocturia, which was present in $19 \%$ cases $(n=356)$, is an important and common presenting complaint. It is a source of significant bother for some patients and is the lower urinary tract symptom most strongly associated with poor quality of life ratings.

Lifestyle changes, (specific diet, fluid and behavioural changes) along with sleep disturbances could be responsible for this symptom.

Nocturia is associated with increased rates of depression, work absenteeism, lower self-rated physical and mental health and increased all-cause mortality. ${ }^{15}$ In the very old, nocturia is associated with higher rates of accidental falls and fractures.

A high-quality Research is essential in order to understand and effectively treat this condition.

About 5\% ( $n=94)$ of the patients complained of thin intermittent urinary stream.

Incidence of recurrent UTI was $26 \%$ and showed a strong correlation with haematuria $(7 \%, \mathrm{n}=131)$ and urgency (34\%, $n=637)$. All those with second infection had either haematuria or urgency or both in past infection. Although, most recurrences are thought to represent reinfections rather than relapse, the reverse occurred in our study. This could be due to improper intake and misuse of antibiotics these days.

Among different types of incontinence, patients with stress urinary incontinence $(20 \%, \mathrm{n}=375)$ exceeded those of urge incontinence $(17 \%, \mathrm{n}=318)$.

Very few patients $(n=3,0.1 \%)$ presented with true incontinence during this study period. Two cases had vesicovaginal fistula and the third one presented with dribbling after hysterectomy. Such low prevalence of true incontinence among patients attending Gynaecology OPD, could be due to their attendance in the existing Urology Department of our Institute. However, the prevalence of female urinary incontinence varies between 5.5\% and 69\% in different surveys. ${ }^{2}$

In the present study, 30\% ( $\mathrm{n}=412)$ had chronic constipation. Liao et al ${ }^{16}$ determined that chronic constipation is a risk factor for many LUTS. Other studies have also reported constipation as a significant risk factor for LUTS. The findings of the present study are compatible with these studies.

Furthermore, an association between constipation and urinary incontinence as well as between constipation and pelvic organ prolapse has been suggested.

The only prospective study in constipated elderly with concomitant LUTS demonstrates that the medical relief of constipation also significantly improves LUTS. Finally, the available data on neuropathic patients suggest that stool impaction in the rectum may mechanically impede bladder emptying.

The other way that constipation can lead to bladder infections is if someone takes too many laxatives to treat the constipation, they can get diarrhoea. Diarrhoeal stool can easily contaminate the perineal area, since the rectum is only a few inches from the urethra. Also, after menopause, there is a decrease in the protective normal vaginal bacteria, so if stool bacteria get into that area it can easily grow. However, the symptoms were least common in older, menopausal women $(1.6 \%, \mathrm{n}=30)$.

All 13 patients with prolapse had chronic constipation with six of them having stress urinary incontinence and seven with increased frequency.

Urinary retention (UR) is a severe impairment of voiding, which can be defined as inability to achieve complete bladder emptying by voluntary micturition. In our study, we came across only 8 patients with urinary retention. The one with acute retention was a young girl of 21 years with a 24 weeks size fibroid uterus and four others were that of chronic urinary retention due to adenomyosis and broad ligament fibroid. The symptoms were relieved after hysterectomy. The remaining three had no demonstrable cause besides severe urinary infection.

The incidence of urinary retention in women is not well documented in literature. One Scandinavian study revealed an incidence of acute urinary retention in women to be 7 per 100,000 population per year.

\section{CONCLUSION}

Symptomatic UTI is very common among sexually active women. LUTS might limit one's daily activity and social life. In the developing world, factors limiting access to care and those which may increase the likelihood of developing UTI result in increased morbidity. Moreover, the antibiotic treatment should be limited to symptomatic UTIs and be initiated after sensitivity testing only. This would avoid drug resistance and also prevent symptomatic cases later with resultant renal damage.

Therefore, studying women's LUTS may be of help in giving proper intervention resources to improve individual's quality of life and also to provide preventive care in the future.

\section{REFERENCES}

[1] Bradway C, Coyne KS, Irwin D, et al. Lower urinary tract symptoms in women-a common but neglected problem. J Am Acad Nurse Pract 2008;20(6):311-8.

[2] Milsom I. Lower urinary tract symptoms in women. Curr Opin Urol 2009;19(4):337-41.

[3] Foxman B, Barlow R, D'Arcy H, et al. Urinary tract infection: self-reported incidence and associated costs. Ann Epidemiol 2000;10(8):509-15.

[4] Mehnert-Kay SA. Diagnosis and management of uncomplicated urinary tract infections. Am Fam Physician 2005; 72(3):451-6.

[5] Medina-Bombardo D, Segui-Diaz M, Roca-Fusalba C, et al. What is the predictive value of urinary symptoms for diagnosing urinary tract infection in women? Fam Pract 2003;20(2)103-07. 


\section{Jemds.com}

[6] Abrams P, Artibani W, Cardozo L, et al. Reviewing the ICS 2002 terminology report: the ongoing debate. Neurourol Urodyn 2009;28(4):287.

[7] Savitha, T. Urinary tract infections. International Journal of Current Research 2011;2(1):67-72.

[8] Mahesh El, Ramesh D, Indumathi VA, et al. Complicated urinary tract infection in a tertiary care center in South India. Al Ameen J Med Sc 2010;3;(2):120-27.

[9] Jollys JV. The reported prevalence of urinary symptoms in women in one rural general practice. $\mathrm{Br} \mathrm{J}$ Gen Pract 1990;40(337):335-7.

[10] Jolleys JV. Factors associated with regular episodes of dysuria among women in one rural general practice. Br J Gen Pract 1991;41(347):241-3.

[11] Sortliffe LD. Urinary tract infections in infants and children. In: Walsh P, Retik AB, Vaughn ED, et al, eds. Campbell's urology. $7^{\text {th }}$ edn. Vol. 2. Philadelphia: WB Saunders 1997:1681-707.

\section{Original Research Article}

[12] EL-Dahr SS, Lewy JE. Urinary tract obstruction and infection in the neonate. Clin Perinatol 1992;19(1):213-22.

[13] Fihn SD, Boyko EJ, Normand EH, et al. Association between use of spermicide-coated condoms and escherichia coli urinary tract infection in young women. Am J Epidemiol 1996;144(5):512-20.

[14] Lomberg $H$, Cedergen $B$, Leffler $H$, et al. Influence of blood group on the availability of receptors for attachment of uropathogenic escherichia coli. Infect Immun 1986;51(3):919-26.

[15] Kupelian V, Fitzgerald MP, Kaplan SA, et al. Association of nocturia and mortality results from the third national health and nutrition examination survey. J Urol 2011;185(2):571-7.

[16] Liao YM, Yang CY, Kao CC, et al. Prevalence and impact on quality of life of lower urinary tract symptoms among a sample of employed women in Taipei: a questionnaire survey. Int J Nurs Stud 2009;46(5):63344. 